\title{
Identification and performance evaluation of housekeeping genes for microRNA expression normalization by reverse transcription-quantitative PCR using liquid-based cervical cytology samples
}

\author{
RHAFAELA LIMA CAUSIN ${ }^{1}$, DANIELLE PESSÔA-PEREIRA ${ }^{1}$, \\ KAREN CRISTINA BORBA SOUZA ${ }^{1}$, ADRIANE FEIJÓ EVANGELISTA ${ }^{1}$, RUI MANUEL VIEIRA REIS ${ }^{1}$, \\ JOSÉ HUMBERTO TAVARES GUERREIRO FREGNANI ${ }^{2}$ and MÁRCIA MARIA CHIQUITELLI MARQUES ${ }^{1,3}$ \\ ${ }^{1}$ Molecular Oncology Research Center, Barretos Cancer Hospital, Barretos, São Paulo 14784-400; \\ ${ }^{2}$ AC Camargo Cancer Center, São Paulo 01509-010; ${ }^{3}$ Barretos School of Health Sciences-FACISB, \\ Barretos, São Paulo 14785-002, Brazil
}

Received April 1, 2019; Accepted July 2, 2019

DOI: $10.3892 / \mathrm{ol} .2019 .10824$

\begin{abstract}
Screening for cervical cancer by cytology has been effective in reducing the worldwide incidence and mortality rates of this disease. However, a number of studies have demonstrated that the sensitivity of conventional cervical cytology may be too low for detection of cervical intraepithelial neoplasias (CIN). Therefore, it is important to incorporate more sensitive molecular diagnostic tests that could substantially improve the detection rates and accuracy for identifying CIN lesions. MicroRNAs (miRNAs) are a class of small non-coding RNAs with the potential to provide robust non-invasive cancer biomarkers for detecting CIN lesions in liquid-based cervical cytology (LBC) samples. At present, there is no consensus on which are the best housekeeping genes for miRNA normalization in LBC. The present study aimed to identify housekeeping genes with consistent and reproducible performance for normalization of reverse transcription-quantitative PCR (RT-qPCR) expression analysis of miRNA using LBC samples. The present study firstly selected six potential candidate housekeeping genes based on a systematic literature evaluation. Subsequently, the expression levels of microRNAs U6, RNU-44, RNU-47, RNU-48, RNU-49 and hsa-miR-16
\end{abstract}

Correspondence to: Dr Márcia Maria Chiquitelli Marques, Molecular Oncology Research Center, Barretos Cancer Hospital, 1331 Rua Antenor Duarte Villela, Barretos, São Paulo 14784-400, Brazil

E-mail: mmcmsilveira@gmail.com

Abbreviations: CC, cervical cancer; miRNA, microRNA; RT-qPCR, reverse transcription-quantitative PCR; Cq, cycle quantification; CIN, cervical intraepithelial neoplasia; HFS, healthy female subjects

Key words: cervical cancer, miRNA expression, liquid-based cytology, housekeeping genes, reverse transcription-quantitative PCR were measured in $40 \mathrm{LBC}$ samples using RT-qPCR. The stability of each potential housekeeping gene was assessed using the NormFinder algorithm. The results revealed that U6 and RNU-49 were the most stable genes among all candidates requiring fewer amplification cycles and smaller variation across the sample set. However, RNU-44, RNU-47, RNU-48 and hsa-miR-16 stability exceeded the recommended housekeeping value suitable for normalization. The findings revealed that U6 may be a reliable housekeeping gene for normalization of miRNA RT-qPCR expression analysis using LBC samples.

\section{Introduction}

Cervical cancer (CC) is the fourth most common cancer among women, with an estimated 527,600 new cases and 265,700 deaths worldwide (1). In developed countries, the incidence and mortality rates have decreased significantly in the past decades. In contrast, the burden from CC remains high in developing and underdeveloped countries due to continuing challenges implementing effective prevention and control programs. Difficulties in less developed countries include barriers to accessing health care services, which are compounded by inadequate cytological examination, usually involving screening with the low coverage of Papanicolaou (Pap) test (2). To implement additional strategies for improving $\mathrm{CC}$ screening, some programs have focused on introducing innovative molecular diagnostic tests such as the molecular HPV testing that could provide more sensitive and specific detection of precursor lesions once validated.

MicroRNAs (miRNAs) are small non-coding RNAs (19 to 24 nucleotides) involved in the post-transcriptional regulation of gene expression (3), where they play a critical role in several cellular processes, such as proliferation, cell growth and apoptosis (4). Many studies have reported aberrant expression of miRNAs in cancer (5), suggesting that these molecules could be used as potential tumor biomarkers. 
Indeed, a recent systematic review identified differentially expressed miRNAs in precursors cervical lesions and CC that could be associated with tumor progression (6). However, most analyses of miRNA expression in $\mathrm{CC}$ have used tumor tissue samples obtained from invasive procedures that cause patient discomfort, such as cervical tissue biopsies or surgery (7). The development of minimally invasive liquid biopsy cytology (LBC) based on miRNA expression (7) is a new approach to identify non-invasive biomarkers for early diagnosis, monitoring response to therapies and for tumor progression (7-9). LBC are considered as an accurate and promising low-cost method for clinical practice but the standardization of miRNA expression analysis remains a major challenge. Thus, standardization of this detection technique is fundamental for the reproducible use of miRNA biomarkers in clinical practice.

Reverse transcription-quantititative PCR (RT-qPCR) is a robust technique frequently used in the diagnosis of many neoplasms and infectious diseases due to its high sensitivity and specificity $(9,10)$. Analysis of miRNAs is considered an important new biomarker because miRNAs are specific and stable in diverse types of clinical samples. However, identification of a constitutively expressed housekeeping genes for adequate normalization of miRNAs expression analysis is a crucial step for better accuracy with this technique. The use of housekeeping genes as endogenous control is the most common method for normalizing RT-qPCR data for miRNA expression (11). Housekeeping genes are internal reaction controls used to gene expression normalization of distinct miRNAs, and they can have different isoforms. For a gene to be considered a reliable housekeeping transcript, it needs to meet some stringent performance criteria (12), such as minimal expression variability between tissues and physiological states of the organism. Moreover, the normalization control being used must faithfully measure any technical variability resulting from differences in the quantity or quality of genetic material being tested (13). Above all else, the most important of these criteria is that the pattern of expression of the normalizer does not interfere or produce artifactual changes in the test samples. Satisfying these basic conditions are the essential properties of a good housekeeping gene for transcript normalization from specific biological samples of interest. The identification of suitable housekeeping genes is a crucial step for deriving reproducible results when investigating the differential expression of miRNAs. The use of unreliable normalization control genes can lead to an incorrect estimate of the expression levels of miRNAs of interest $(14,15)$. For this reason, the choice of appropriate housekeeping genes for normalizing the expression of miRNAs analysis using LBC is an important issue to be solved. No housekeeping gene is unique and constitutively expressed in all sample types, as well as different types of diseases in all experimental designs, which indicates that the stability of housekeeping gene expression should be checked rigorously $(16,17)$.

This is the first study to evaluate control housekeeping genes for miRNA RT-qPCR data normalization in LBC cervical samples. Since there is little consensus on the best choice of normalizers, we performed a literature review to identify housekeeping genes most commonly used in miRNA RT-qPCR data normalization. In addition, we evaluated their relative expression levels in $\mathrm{LBC}$ samples from patients who underwent routine cervical cancer screening.

\section{Materials and methods}

Study design. In order to select suitable housekeeping genes, we conducted a systematic two-phase analysis including an initial exploratory review of the literature, followed by a laboratory evaluation phase of selected genes (Fig. 1).

Phase 1, exploratory phase. We first performed a PubMed systematic literature review to identify candidate housekeeping genes for miRNA normalization in RT-qPCR using the following keywords: Cervical cancer; miRNA expression, RT-qPCR. Inclusion criteria were defined as follows: i) Original articles; ii) the language in which the article should be published was English; iii) studies that only used precursor lesions or CC samples; iv) studies that performed miRNA expression for RT-qPCR. We found a total of 109 articles, which were available at the end of March 2018. Of these, 70 studies (10-79) were eligible for inclusion in our selection of candidate housekeeping genes (Table I).

Phase 2, evaluation phase. All candidate housekeeping genes identified by our literature review were selected for expression analysis by RT-qPCR and tested in the LBC samples from 5 CC (5), 20 CIN (5 CIN3; 5 CIN2 and 10 CIN1) and 15 healthy women (HSF-without CIN). We considered a housekeeping gene to be suitable for normalization purposes when it was stably expressed across all samples independently of the histological condition, and when the cycle quantification $(\mathrm{Cq})$ values did not exceed 35 . Finally, the best housekeeping gene was evaluated using the NormFinder algorithm, which is software designed to identify the optimal normalization gene among a set of candidates (80).

Study population and sample collection. We analyzed a total of 40 LBC samples randomly obtained from women who had undergone routine colposcopy in the Department of Prevention of the Barretos Cancer Hospital in 2014. All samples were collected immediately before colposcopy and preserved in ThinPrep ${ }^{\mathrm{TM}}$ Pap test (Hologic) for subsequent molecular analyses. ThinPrep ${ }^{\mathrm{TM}}$ samples were classified into five groups: HFS; low-grade CIN (CIN1); high-grade CIN (CIN2 group or CIN3 group); and CC. All $\mathrm{CIN}$ - and CC-histological diagnoses from women who presented with suspicious/abnormal areas during colposcopy were subsequently confirmed by analyses of tissue samples collected for the Department of Pathology of the Barretos Cancer Hospital using a colposcopy-guided cervical biopsy.

RNA isolation. ThinPrep ${ }^{\mathrm{TM}}$ samples were manually washed to remove the buffered preservative solution and to lyse blood cells, which could inhibit downstream molecular analyses. Total RNA was performed using the RecoverAll Total Nucleic Acid Isolation Kit (Thermo Fisher Scientific), according to the manufacturer's protocol. The purity of total RNA was evaluated by NanoDrop ${ }^{\circledR}$ Spectrophotometer v3.7 (Thermo Fisher Scientific).

$R T-q P C R$. Considering that the focus of this study is LBC cervical samples we do not use housekeeping genes to analyze 


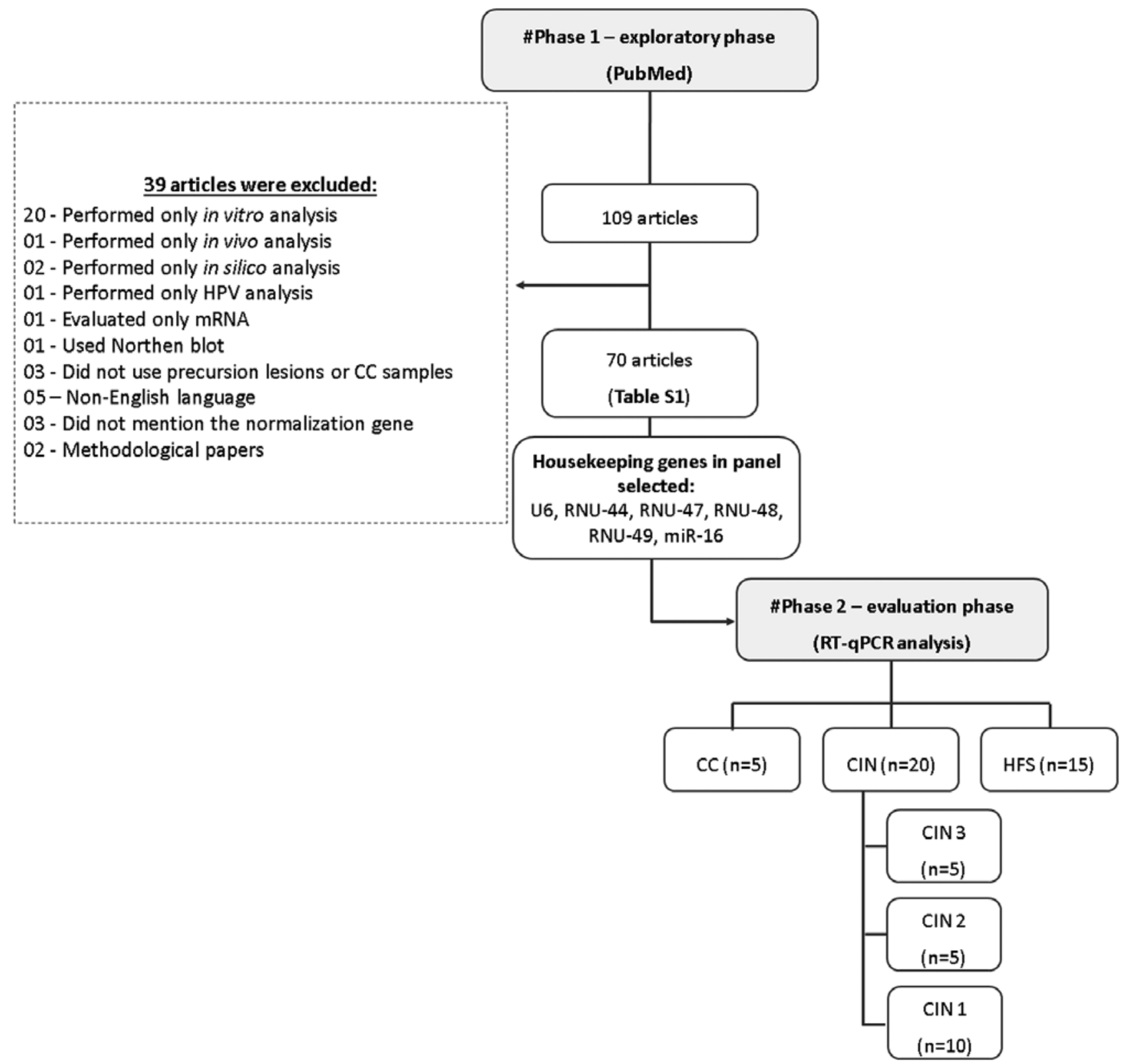

Figure 1. Flowchart depicting the exploratory and evaluation phases of selection of suitable housekeeping genes for normalization. CC, cervical cancer; CIN, cervical intraepithelial neoplasia; HFS, healthy female subjects; RT-qPCR, reverse transcription-quantitative PCR.

the expression of these miRNAs in tissue samples. To perform RT-qPCR reactions we used TaqMan microRNA assays (Thermo Fisher Scientific) using LBC cervical samples. Initially, a target-specific stem-loop reverse transcription RT-PCR was performed using a High Capacity cDNA Reverse Transcription kit (Thermo Fisher Scientific), following the protocol provided by the manufacturer. Briefly, for each sample $10 \mathrm{ng}$ of total RNA was reverse transcribed using miRNA-specific primers and TaqMan Assays (Table SI) in a $15 \mu \mathrm{l}$ reaction volume for $30 \mathrm{~min}$ at $16^{\circ} \mathrm{C}, 20 \mathrm{~min}$ at $42^{\circ} \mathrm{C}$ and $5 \mathrm{~min}$ at $85^{\circ} \mathrm{C}$. All RT-PCR reactions were performed using the Proflex ${ }^{\mathrm{TM}} 3 \mathrm{x} 32$-well PCR system (Thermo Fisher Scientific). Then $2 \mu \mathrm{l}$ of the reverse transcription products (cDNA) was amplified in the QuantStudio 6 Flex Real-Time PCR system (Thermo Fisher Scientific) using the TaqMan Universal PCR Master Mix II (Thermo Fisher Scientific), according to the manufacturer's protocol. All RT-qPCR reactions were performed in triplicate using
Taqman probes. The PCR protocol comprised 40 cycles of $2 \mathrm{~min}$ at $50^{\circ} \mathrm{C}, 10 \mathrm{~min}$ at $95^{\circ} \mathrm{C}, 15 \mathrm{sec}$ at $95^{\circ} \mathrm{C}$ and $1 \mathrm{~min}$ at $60^{\circ} \mathrm{C}$. The Threshold Cycle $(\mathrm{Cq})$ values were determined using the same threshold setting and analyzed according to a previously reported method (81).

NormFinder-based stability analysis. Comparison between expression values was performed using NormFinder algorithm (80), an ANOVA model-based approach which estimates intragroup and inter-group expression variations in order to calculate stability values for each candidate gene (18). We used exponentially transformed $\mathrm{Cq}$ values $\left(2^{-\mathrm{Cq}}\right)$ as input data in the NormFinder software, which ranks genes based on their expression stability in a given sample set with a specific experimental design. The lowest value calculated by the software indicates the most stable endogenously expressed gene for optimal normalization. 
Table I. Bibliographic survey of reference genes.

\begin{tabular}{|c|c|c|c|}
\hline Author, year & Reference gene & Sample & (Refs.) \\
\hline $\begin{array}{l}\text { Yao et al, 2013; Yang et al, 2015; Yu et al, 2014; Wang et al, 2013; } \\
\text { Yin et al, 2015; Wang et al, 2015; Song et al, 2015; Li et al, 2015; } \\
\text { Wei et al, 2012; Chen et al, 2013; Yue et al, 2011; Zhou et al, 2016; } \\
\text { Chen and Liu, 2016; Sun et al, 2016; Zhang et al, 2016; } \\
\text { Xiao et al, 2014; Yi et al, 2016; Cheng et al, 2016; } \\
\text { Sun et al, 2017; Yu et al, 2013; Yang et al, 2015; Qin et al, 2015; } \\
\text { Sun et al, 2017; Chandrasekaran et al, 2016; Lin } \text { et al, 2017; } \\
\text { Azizmohammadi et al, 2017; Jin et al, 2017; Liu and Ni, 2018; } \\
\text { Zhou et al, 2017; Song et al, 2017; Zhao et al, 2017; } \\
\text { He et al, 2017; Li et al, 2017; Hu et al, 2017; Luo et al, 2017; } \\
\text { Zhang et al, 2018; Li et al, 2017; Zhao et al, 2017; Tao et al, } 2017\end{array}$ & U6 & Tissue & $(10-48)$ \\
\hline $\begin{array}{l}\text { Gocze et al, 2015; Zhang et al, 2013; Chen et al, 2014; } \\
\text { Wang et al, 2015; Zeng et al, 2015; Ma et al, 2015; } \\
\text { Zheng et al, 2015; Zhang et al, } 2016\end{array}$ & U6 & FFPE & $(49-57)$ \\
\hline Zhao et al, 2013; Jia et al, 2015; Jiang et al, 2017; Zhou et al, 2017 & U6 & Serum & $(58-61)$ \\
\hline Yu et al, 2012 & U6 & Blood & $(62)$ \\
\hline $\begin{array}{l}\text { Chen et al, 2013; Hu et al, 2017; You et al, 2015; Luo et al, 2015; } \\
\text { Liu et al, 2015; Xie et al, 2015; Shen et al, 2013; Huang et al, } 2012\end{array}$ & RNU6B & Tissue & $(19,43,63-68)$ \\
\hline Xing et al, 2013; Lee et al, 2014 & RNU6B & FFPE & $(69,70)$ \\
\hline Ivanov et al, 2018 & U6 & $\begin{array}{l}\text { Air-dried cervical } \\
\text { PAP smears }\end{array}$ & $(71)$ \\
\hline Gocze et al, 2015; Yu et al, 2016; Hao et al, 2016 & $5 \mathrm{~S}$ & FFPE & $(49,72,73)$ \\
\hline Shen et al, 2013 & $18 \mathrm{~S}$ & Tissue & $(66)$ \\
\hline Sun et al, 2017; Nagamitsu et al, 2016 & $\operatorname{miR}-16$ & Serum & $(74,28)$ \\
\hline Kogo et al, 2015; Lajer et al, 2012 & RNU44 & Tissue & $(75,76)$ \\
\hline Chen et al, 2013 & RNU44 & Serum & (19) \\
\hline Huang et al, 2014; Myklebust et al, 2011 & RNU48 & Tissue & $(77,78)$ \\
\hline Chen et al, 2013 & RNU48 & Serum & $(19)$ \\
\hline Sharma et al, 2016 & $\operatorname{miR}-127$ & Tissue & $(79)$ \\
\hline
\end{tabular}

FFPE, formalin-fixed paraffin-embedded.

Statistical analysis. All variables were presented using mean values and standard deviation (SD). ANOVA with a Bonferroni post hoc test and the Kruskall-Wallis tests were used to compare the mean values of continuous variables across the histologic groups. P-values of $<0.05$ were considered statistically significant. All statistical analyses were performed with SPSS for Windows, v.21.0 (IBM Corporation). All graphs was expressed just descriptive analysis data.

\section{Results}

We selected six candidate housekeeping genes based on their expression profile across the reviewed studies (Table I): U6 (U6 small nuclear RNA); miR-16 (hsa-microRNA-16); RNU-44 (SNORD44 small nucleolar RNA); RNU-48 (SNORD48 small nucleolar RNA); RNU-47 (SNORD47 small nucleolar RNA); and RNU-49 (SNORD49A small nucleolar RNA). Most of the candidate genes have previously been described as housekeeping transcripts for miRNA normalization for expression quantification using different types of biological samples, such as fresh tissue biopsies, formalin-fixed paraffin-embedded (FFPE) tissues, serum or plasma, and air-dried cervical smears. Of the six selected housekeeping genes, only miR-16 has previously been used as an endogenous control for LBC expression analysis $(28,74)$.

The technical performance of six selected candidate housekeeping genes as normalization controls was investigated using 40 LBC samples. We found that U6 and RNU-49 had the lowest $\mathrm{Cq}$ value variation among the six tested candidate housekeeping genes (Fig. 2). Furthermore, both genes amplified more efficiently than the other candidates and required fewer amplification cycles to achieve $\mathrm{Cq}$ values above background fluorescence levels. The lower $\mathrm{Cq}$ value for U6 $(21,81)$ indicated that their expression levels and PCR efficiencies required fewer cycles of amplification to reach the detection threshold. In addition, we found that the U6 gene was more uniformly expressed in LBC samples than the other candidate housekeeping genes. In contrast, more cycles of amplification were required (Cq values $>35$ ) for RNU-44, RNU-47, RNU-48 e miR-16, indicating that these genes might 


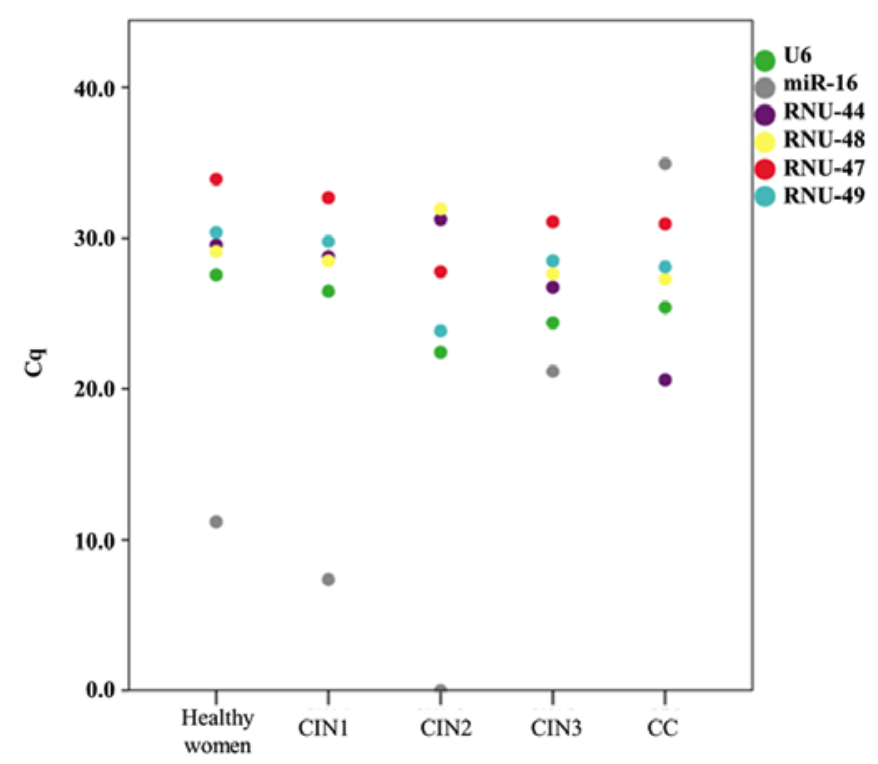

Figure 2. Distribution of mean $\mathrm{Cq}$ values according to histologic groups. CIN, cervical intraepithelial neoplasia; $\mathrm{Cq}$, cycle quantification; $\mathrm{CC}$, cervical cancer.

not be suitable housekeeping genes for normalization using LBC samples.

Candidate housekeeping gene expression according histological groups. To assess whether the candidate housekeeping genes were differentially expressed in varying histologic conditions, we compared the mean $\mathrm{Cq}$ values of the candidate housekeeping genes obtained from each of five histologic groups (Table II). We found no significant differences in the expression of U6 (P-value, 0.06) and RNU-49 (P-value: 0.128) across all groups, supporting their stable performance and potential as robust endogenous controls in RT-qPCR normalization using LBC samples. We did not find a significant association for RNU-47 (P-value, 0.064) and this gene required a greater number of amplification cycles across the experimental groups, especially in LBC samples from patients HFS (34.16 \pm 2.85$)$. We also observed significant differences for miR-16 (P-value, 0.045); RNU-44 (P-value, 0.004); and RNU-48 (P-value, 0.022), indicating that these genes can be differentially expressed in LBC samples from patients with different cervical histology and that such variation could lead to inconsistent normalization. Indeed, miR-16 exhibited the most variable expression across the groups (Fig. 3), ranging from no amplification at all in LBC samples from CIN2 patients to low levels in CIN1 patients (7.35 \pm 15.51$)$. Furthermore, RNU-44 and RNU-48 were more abundant in LBC samples from CC patients (mean Cq values: $20.60 \pm 11.60$ and $27.27 \pm 2.30$, respectively) in comparison to other histologic groups indicating that they would likely bias expression values.

Expression stability analysis. We further analyzed the stability values of each candidate housekeeping gene using the NormFinder algorithm. We found that among the six candidates, U6 was the most stable gene (stability value, 0.856), followed by RNU-49 (0.929) (Fig. 4). In contrast, the other candidate housekeeping genes presented inadequate stability values, ranging from 2.375 to 12.901 . These findings suggested that U6 and RNU-49 were the best housekeeping genes in LBC samples, whereas miR-16, RNU-44, RNU-47, and RNU-48 should not be considered suitable for use as endogenous controls for RT-qPCR normalization.

\section{Discussion}

There have been several studies investigating the utility of miRNA in translational research, considering deregulated expression in diverse diseases, variation in tissue-specific distribution and the overall stability of miRNA in different clinical samples (19-22). Indeed, there is emerging evidence demonstrating the feasibility of using miRNAs as non- or minimally invasive diagnostic biomarkers in cancer. For instance, Rossi et al (82) evaluated a five-miRNA expression signature developed for thyroid lesions using fine needle aspiration cytology (FNAC). Their analysis suggested miR-375 as a promising preoperative biomarker for distinguishing benign from malignant follicular neoplasms. In another study, Kottaridi et al (83) designed a panel of seven overexpressed miRNAs for use in histologically confirmed LBC malignant endometrial samples to discriminate between non-malignant and malignant specimens and to identify any samples with inadequate RNA. There are many studies that have reported promising molecular approaches to LBC samples for clinical laboratories (84-86). None of the studies to date have focused on evaluating miRNAs in LBC cervical samples, which could be considered an important minimally invasive approach for cancer detection by miRNA expression data. For this reason, RT-qPCR is now one of the most commonly used new methods for the evaluation of miRNA expression due to its high sensitivity and reproducibility $(87,88)$.

Since reliable normalization is fundamental to RT-qPCR, there is a need to choose a suitable gene for use as an endogenous control in order to obtain an accurate miRNA expression and to ensure consistency. The selection of housekeeping genes as normalizers for miRNA has relied on choosing from distinct miRNAs and other small RNAs, such as U6, RNU6B, miR-16, and RNU-44 $(37,68,76,82)$. However, the choice of housekeeping gene remains quite empirical because, to the best of our knowledge, there are no previous studies that have validated endogenous housekeeping control genes for miRNA normalization in LBC cervical samples.

In this study, we evaluated six candidate housekeeping genes for miRNA RT-qPCR data using LBC samples from patients who underwent cervical cancer screening. We analyzed the expression of five small nucleolar (sno) RNAs: RNU-44, RNU-47, RNU-48, RNU-49 and U6. The snoRNAs are a group of non-coding RNAs with variable length (80 to $1000 \mathrm{nt}$ in yeast), mainly required for ribosomal RNA (rRNA) maturation (89). Many types of snoRNAs have been described in eukaryotes and each of them corresponds to a specific mode of transcription (90) and have been used as housekeeping genes for miRNA normalization $(30,31)$. Some studies that have used miRNA profiling to discriminate cervical cancer from benign lesions selected RNU-44 and RNU-48 as endogenous controls for normalization of miRNA RT-qPCR data, mostly using tissue $(26,32-34)$ and serum samples (35). However, our findings suggest that these snoRNAs are unsuitable for miRNA 
Table II. Association between mean Cq values and histologic groups.

\begin{tabular}{lcccccc}
\hline Housekeeping genes & HFS & CIN 1 & CIN 2 & CIN 3 & CC & P-value \\
\hline U6 & $27.76 \pm 0.48$ & $26.47 \pm 1.38$ & $22.44 \pm 12.71$ & $24.40 \pm 1.77$ & $25.41 \pm 1.97$ & 0.060 \\
miR-16 & $11.19 \pm 17.49$ & $7.35 \pm 15.51$ & - & $21.16 \pm 19.34$ & $34.94 \pm 1.94$ & $0.045^{\text {a }}$ \\
RNU-44 & $30.03 \pm 3.49$ & $28.78 \pm 2.06$ & $31.25 \pm 2.41$ & $26.74 \pm 1.47$ & $20.60 \pm 11.60$ & $0.004^{\mathrm{a}}$ \\
RNU-47 & $34.16 \pm 2.85$ & $32.68 \pm 2.85$ & $27.78 \pm 15.58$ & $31.09 \pm 1.62$ & $30.94 \pm 1.80$ & 0.064 \\
RNU-48 & $29.45 \pm 2.72$ & $28.51 \pm 2.14$ & $31.94 \pm 2.08$ & $27.64 \pm 1.77$ & $27.27 \pm 2.30$ & $0.022^{\text {a }}$ \\
RNU-49 & $30.67 \pm 2.73$ & $29.77 \pm 2.07$ & $23.84 \pm 13.34$ & $28.51 \pm 1.03$ & $28.08 \pm 0.95$ & 0.128
\end{tabular}

-, no amplification. ${ }^{\mathrm{a}} \mathrm{P}<0.05$. CC, cervical cancer; $\mathrm{CIN}$, cervical intraepithelial neoplasia; $\mathrm{Cq}$, cycle quantification; HFS, healthy female subjects .

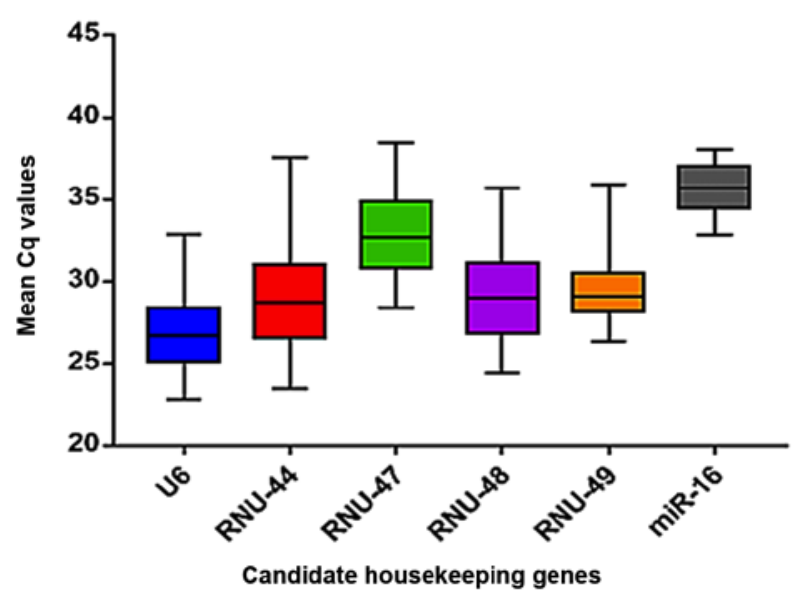

Figure 3. Box plots showing the distribution of mean $\mathrm{Cq}$ values of the six candidate housekeeping genes. $\mathrm{Cq}$, cycle quantification.

normalization in LBC samples, due to the higher number of amplification cycles required and the differential expression across distinct histologic groups. In addition, RNU-47 also required more amplification, confirming that it may not be an appropriate housekeeping gene. In contrast, RNU-49 and U6 could be amplified with fewer cycles and both have smaller variation according to NormFinder algorithm. Several studies have used U6 as housekeeping gene for RT-qPCR data normalization in cervical tissues (10-13), whole blood (62), serum $(19,58,59)$ and air-dried Pap smears $(71)$, but not previously in LBC samples. In agreement with other studies, our analyses indicate that U6 is the best housekeeping gene for LBC samples.

We also evaluated the miR-16 expression in LBC samples because it has been suggested as a housekeeping gene for cervical samples in other studies $(25,45)$. There are doubts about the reliability of this gene for normalization because some studies have reported miR-16 as differentially expressed in CC. Zubillaga-Guerrero et al (91) demonstrated altered expression of miR-16 in CC, with miR-16 downregulating cyclin E1 (CCNE1) gene expression in cervical cancer cell lines. These data suggest a potential role of miR-16 in modulating cell cycle in CC and make it less likely to be a suitable control housekeeping gene. A recent systematic review also shown that miR-16 was deregulated and associated with cervical cancer progression (6). In our study, we confirmed

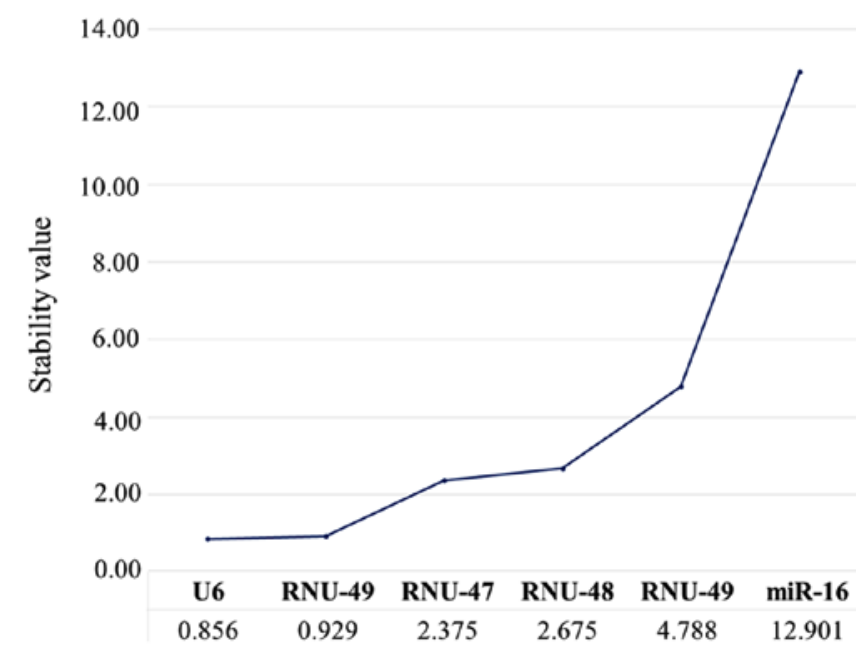

Figure 4. Stability values obtained using the NormFinder algorithm.

that miR-16 was not a good endogenous control for RT-qPCR normalization in LBC samples because it presented higher variability expression across all samples-including amplification under background fluorescence in some cases-and altered expression in different histologic conditions.

In summary, our data demonstrates that U6 and RNU-49 are suitable housekeeping genes that can be used for miRNA RT-qPCR analyzes in LBC samples from patients who underwent cervical cancer screening. This is the first study that provide comprehensive information on the analytical performance of these genes for future normalizations of miRNA expression studies in LBC cervical samples that can be very useful for application of miRNAs in screening with LBC and had a clinical significance. However, future studies using RT-qPCR may to demonstrate in practice housekeepings U6 and RNU-49 may be excellent normalizers for liquid-based cytology (LBC) cervical samples. Thus, continued research efforts should be made about miRNA expression analysis wisely differentially expressed in these histological subtypes and LBC cervical samples using other tools for the stability analysis of housekeepings, such as geNorm.

\section{Acknowledgements}

The authors would like to thank Dr Jeremy Squire (Genetics Department, University of Sao Paulo, Ribeirão Preto, São 
Paulo, Brazil) for carefully proofreading the manuscript and for providing constructive criticism.

\section{Funding}

The present study was supported by internal funding from FAPESP (grant no. 2016/15831-3) and the Public Ministry of Work.

\section{Availability of data and materials}

The datasets used and/or analyzed during the current study are available from the corresponding author on reasonable request.

\section{Authors' contributions}

RLC developed and led the overall study, conducted the data reviews and the analysis, and prepared the manuscript. DPP participated in setting up the PCR assay and prepared the manuscript. KCBS participated in setting up the PCR assay and critically revised the manuscript. AFE, RMVR and JHTGF designed and developed the study, and critically revised the manuscript. MMCMS conceived the study, provided advice during the study development and prepared the manuscript.

\section{Ethics approval and consent to participate}

The present study was approved by the Research Ethics Committee of the Barretos Cancer Hospital (approval no. 784/2014). Each research participant provided written informed consent for the publication of any data associated with the present study. All information that could be used to identify the study participants was kept confidential and encrypted in a secure database to ensure full confidentiality of clinical information, laboratory findings and the anonymity of each participant.

\section{Patient consent for publication}

Not applicable.

\section{Competing interests}

The authors declare that they have no competing interests.

\section{References}

1. Torre LA, Bray F, Siegel RL, Ferlay J, Lortet-Tieulent J and Jemal A: Global cancer statistics, 2012. CA Cancer J Clin 65 : 87-108, 2015

2. Parkin DM and Bray F: The burden of HPV-related cancers. Vaccine 24 (Suppl 3): S11-S25, 2006.

3. Iorio MV and Croce CM: Causes and consequences of microRNA dysregulation. Cancer J 18: 215-222, 2012.

4. He Y, Lin J, Ding Y, Liu G, Luo Y, Huang M, Xu C, Kim TK, Etheridge A, Lin M, et al: A systematic study on dysregulated microRNAs in cervical cancer development. Int J Cancer 138 1312-1327, 2016.

5. He L and Hannon GJ: MicroRNAs: Small RNAs with a big role in gene regulation. Nat Rev Genet 5: 522-531, 2004.

6. Pardini B, De Maria D, Francavilla A, Di Gaetano C, Ronco G and Naccarati A: MicroRNAs as markers of progression in cervical cancer: A systematic review. BMC Cancer 18: 696, 2018

7. Di Meo A, Bartlett J, Cheng Y, Pasic MD and Yousef GM: Liquid biopsy: A step forward towards precision medicine in urologic malignancies. Mol Cancer 16, 80, 2017.
8. Finotti A, Allegretti M, Gasparello J, Giacomini P, Spandidos DA, Spoto G and Gambari R: Liquid biopsy and PCR-free ultrasensitive detection systems in oncology (Review). Int J Oncol 53: 1395-1434, 2018

9. Muinelo-Romay L, Casas-Arozamena C and Abal M: Liquid biopsy in endometrial cancer: New opportunities for personalized oncology. Int J Mol Sci 19: E2311, 2018.

10. Yao T, Rao Q, Liu L, Zheng C, Xie Q, Liang J and Lin Z: Exploration of tumor-suppressive microRNAs silenced by DNA hypermethylation in cervical cancer. Virol J 10: 175, 2013.

11. Yang Y, Xie YJ, Xu Q, Chen JX, Shan NC and Zhang Y: Down-regulation of miR-1246 in cervical cancer tissues and its clinical significance. Gynecol Oncol 138: 683-688, 2015.

12. Yu Q, Liu SL, Wang H, Shi G, Yang P and Chen XL: miR-126 Suppresses the proliferation of cervical cancer cells and alters cell sensitivity to the chemotherapeutic drug bleomycin. Asian Pac J Cancer Prev 14: 6569-6572, 2014.

13. Wang L, Wang Q, Li HL and Han LY: Expression of MiR200a, miR93, metastasis-related gene RECK and MMP2/MMP9 in human cervical carcinoma-relationship with prognosis. Asian Pac J Cancer Prev 14: 2113-2118, 2013.

14. Yin ZL, Wang YL, Ge SF, Guo TT, Wang L, Zheng XM and Liu J: Reduced expression of miR-503 is associated with poor prognosis in cervical cancer. Eur Rev Med Pharmacol Sci 19: 4081-4085, 2015.

15. Wang LQ, Zhang Y, Yan H, Liu KJ and Zhang S: MicroRNA-373 functions as an oncogene and targets YOD1 gene in cervical cancer. Biochem Biophys Res Commun 459: 515-520, 2015.

16. Song X, Shi B, Huang K and Zhang W: miR-133a inhibits cervical cancer growth by targeting EGFR. Oncol Rep 34: 1573-1580, 2015.

17. Li QQ, Zhang L, Wan HY, Liu M, Li X and Tang H: CREB1-driven expression of miR-320a promotes mitophagy by down-regulating VDAC1 expression during serum starvation in cervical cancer cells. Oncotarget 6: 34924-34940, 2015.

18. Wei Q, Li YX, Liu M, Li X and Tang H: MiR-17-5p targets TP53INP1 and regulates cell proliferation and apoptosis of cervical cancer cells. IUBMB Life 64: 697-704, 2012.

19. Chen J, Yao D, Li Y, Chen H, He C, Ding N, Lu Y, Ou T, Zhao S, $\mathrm{Li} \mathrm{L}$ and Long F: Serum microRNA expression levels can predict lymph node metastasis in patients with early-stage cervical squamous cell carcinoma. Int J Mol Med 32: 557-567, 2013.

20. Yue C, Wang M, Ding B, Wang W, Fu S, Zhou D, Zhang Z and Han S: Polymorphism of the pre-miR-146a is associated with risk of cervical cancer in a Chinese population. Gynecol Oncol 122: 33-37, 2011.

21. Zhou Q, Han LR, Zhou YX and Li Y: MiR-195 suppresses cervical cancer migration and invasion through targeting Smad3. Int J Gynecol Cancer 26: 817-824, 2016.

22. Chen XF and Liu Y: MicroRNA-744 inhibited cervical cancer growth and progression through apoptosis induction by regulating Bcl-2. Biomed Pharmacother 81: 379-387, 2016.

23. Sun Y, Yang X, Liu M and Tang H: B4GALT3 up-regulation by miR-27a contributes to the oncogenic activity in human cervical cancer cells. Cancer Lett 375: 284-292, 2016.

24. Zhang J, Wang F, Xu J, Wang X, Ye F and Xie X: Micro ribonucleic acid-93 promotes oncogenesis of cervical cancer by targeting RAB11 family interacting protein 1. J Obstet Gynaecol Res 42: 1168-1179, 2016.

25. Xiao S, Liao S, Zhou Y, Jiang B, Li Y and Xue M: High expression of octamer transcription factor 1 in cervical cancer. Oncol Lett 7: 1889-1894, 2014.

26. Yi Y, Li H, Lv Q, Wu K, Zhang W, Zhang J, Zhu D, Liu Q and Zhang W: miR-202 inhibits the progression of human cervical cancer through inhibition of cyclin D1. Oncotarget 7: 72067-72075, 2016.

27. Cheng YX, Zhang QF, Hong L, Pan F, Huang JL, Li BS and Hu M: MicroRNA-200b suppresses cell invasion and metastasis by inhibiting the epithelial-mesenchymal transition in cervical carcinoma. Mol Med Rep 13: 3155-3160, 2016.

28. Sun L, Jiang R, Li J, Wang B, Ma C, Lv Y and Mu N: MicoRNA-425-5p is a potential prognostic biomarker for cervical cancer. Ann Clin Biochem 54: 127-133, 2017.

29. Yu Y, Zhang Y and Zhang S: MicroRNA-92 regulates cervical tumorigenesis and its expression is upregulated by human papillomavirus-16 E6 in cervical cancer cells. Oncol Lett 6: 468-474, 2013.

30. Yang Y, Liu H, Wang X and Chen L: Up-regulation of microRNA-664 inhibits cell growth and increases cisplatin sensitivity in cervical cancer. Int J Clin Exp Med 8: 18123-18129, 2015.

31. Qin X, Wan Y, Wang S and Xue M: MicroRNA-125a-5p modulates human cervical carcinoma proliferation and migration by targeting ABL2. Drug Des Devel Ther 10: 71-79, 2015. 
32. Sun P, Shen Y, Gong JM, Zhou LL, Sheng JH and Duan FJ: A new MicroRNA expression signature for cervical cancer. Int J Gynecol Cancer 27: 339-343, 2017.

33. Chandrasekaran KS, Sathyanarayanan A and Karunagaran D: MicroRNA-214 suppresses growth, migration and invasion through a novel target, high mobility group AT-hook 1, in human cervical and colorectal cancer cells. Br J Cancer 115: 741-751, 2016.

34. Lin W, Feng M, Chen G, Zhou Z, Li J and Ye Y: Characterization of the microRNA profile in early-stage cervical squamous cell carcinoma by next-generation sequencing. Oncol Rep 37: 1477-1486, 2017.

35. Azizmohammadi S, Safari A, Azizmohammadi S, Kaghazian M, Sadrkhanlo M, Yahaghi E, Farshgar R and Seifoleslami M: Molecular identification of miR-145 and miR-9 expression level as prognostic biomarkers for early-stage cervical cancer detection. QJM 110: 11-15, 2017.

36. Jin X, Chen X, Hu Y, Ying F, Zou R, Lin F, Shi Z, Zhu X, Yan X, Li S and Zhu H: LncRNA-TCONS 00026907 is involved in the progression and prognosis of cervical cancer through inhibiting miR-143-5p. Cancer Med 6: 1409-1423, 2017.

37. Liu J and Ni S: Association between genetic polymorphisms in the promoters of let-7 and risk of cervical squamous cell carcinoma. Gene 642: 256-260, 2018

38. Zhou Y, Hao Y, Li Y, Li R, Wu R, Wang S and Fang Z: Amplification and up-regulation of MIR30D was associated with disease progression of cervical squamous cell carcinomas. BMC Cancer 17: 230, 2017.

39. Song R, Cong L, Ni G, Chen M, Sun H, Sun Y and Chen M: MicroRNA-195 inhibits the behavior of cervical cancer tumors by directly targeting HDGF. Oncol Lett 14: 767-775, 2017.

40. Zhao J, Li B, Shu C, Ma Y and Gong Y: Downregulation of miR-30a is associated with proliferation and invasion via targeting MEF2D in cervical cancer. Oncol Lett 14: 7437-7442, 2017.

41. He S, Liao B, Deng Y, Su C, Tuo J, Liu J, Yao S and Xu L: MiR-216b inhibits cell proliferation by targeting FOXM1 in cervical cancer cells and is associated with better prognosis. BMC Cancer 17: 673, 2017.

42. Li S, Yang F, Wang M, Cao W and Yang Z: miR-378 functions as an onco-miRNA by targeting the ST7L/Wnt/ $\beta$-catenin pathway in cervical cancer. Int J Mol Med 40: 1047-1056, 2017.

43. Hu Y, Xie H, Liu Y, Liu W, Liu M and Tang H: miR-484 suppresses proliferation and epithelial-mesenchymal transition by targeting ZEB1 and SMAD2 in cervical cancer cells. Cancer Cell Int 17: 36, 2017

44. Luo S,LiN, Yu S, Chen L, Liu C and Rong J: MicroRNA-92a promotes cell viability and invasion in cervical cancer via directly targeting Dickkopf-related protein 3. Exp Ther Med 14: 1227-1234, 2017.

45. Zhang Z, Wang J, Wang X, Song W, Shi Y and Zhang L: MicroRNA-21 promotes proliferation, migration, and invasion of cervical cancer through targeting TIMP3. Arch Gynecol Obstet 297: 433-442, 2018.

46. Li M,Li BY,Xia H and Jiang LL: Expression of microRNA-142-3p in cervical cancer and its correlation with prognosis. Eur Rev Med Pharmacol Sci 21: 2346-2350, 2017.

47. Zhao Y, Liu X and Lu YX: MicroRNA-143 regulates the proliferation and apoptosis of cervical cancer cells by targeting HIF-1 $\alpha$. Eur Rev Med Pharmacol Sci 21: 5580-5586, 2017.

48. Tao L, Zhang CY, Guo L, Li X, Han NN, Zhou Q and Liu ZL: MicroRNA-497 Accelerates Apoptosis While Inhibiting Proliferation, Migration, and Invasion through negative regulation of the MAPK/ERK signaling pathway via RAF-1. J Cell Physiol 233: 6578-6588, 2018.

49. Gocze K, Gombos K, Kovacs K, Juhasz K, Gocze P and Kiss I: MicroRNA expressions in HPV-induced cervical dysplasia and cancer. Anticancer Res 35: 523-530, 2015.

50. Zhang J, Zheng F, Yu G, Yin Y and Lu Q: miR-196a targets netrin 4 and regulates cell proliferation and migration of cervical cancer cells. Biochem Biophys Res Commun 440: 582-588, 2013

51. Chen Y, Ma C, Zhang W, Chen Z and Ma L: Down regulation of miR-143 is related with tumor size, lymph node metastasis and HPV16 infection in cervical squamous cancer. Diagn Pathol 9: 88, 2014.

52. Wang $C$ and Jiang T: MicroRNA-335 represents an independent prognostic marker in cervical cancer. Tumour Biol 36: 5825-5830, 2015.

53. Zeng K, Zheng W, Mo X, Liu F, Li M, Liu Z, Zhang W and Hu X: Dysregulated microRNAs involved in the progression of cervical neoplasm. Arch Gynecol Obstet 292: 905-913, 2015

54. Ma L, Hong Y, Lu C, Chen Y and Ma C: The occurrence of cervical cancer in Uygur women in Xinjiang Uygur Autonomous Region is correlated to microRNA-146a and ethnic factor. Int J Clin Exp Pathol 8: 9368-9375, 2015.
55. Zheng W, Liu Z, Zhang W and $\mathrm{Hu} \mathrm{X}$ : miR-31 functions as an oncogene in cervical cancer. Arch Gynecol Obstet 292: 1083-1089, 2015.

56. Qian K, Pietilä T, Rönty M, Michon F, Frilander MJ, Ritari J, Tarkkanen J, Paulín L, Auvinen P and Auvinen E: Identification and Validation of Human Papillomavirus Encoded microRNAs. PLoS One 8: e70202 2013.

57. Zhang J, Jia J, Zhao L, Li X, Xie Q, Chen X, Wang J and Lu F: Down-regulation of microRNA-9 leads to activation of IL-6/Jak/STAT3 pathway through directly targeting IL-6 in HeLa cell. Mol Carcinog 55: 732-742, 2016.

58. Zhao S, Yao D, Chen J and Ding N: Circulating miRNA-20a and miRNA-203 for screening lymph node metastasis in early stage cervical cancer. Genet Test Mol Biomark 17: 631-636, 2013.

59. Jia W, Wu Y,Zhang Q, Gao GE, Zhang C and Xiang Y: Expression profile of circulating microRNAs as a promising fingerprint for cervical cancer diagnosis and monitoring. Mol Clin Oncol 3: 851-858, 2015.

60. Jiang W,Pan JJ,Deng YH,Liang MR and YaoLH:Down-regulated serum microRNA-101 is associated with aggressive progression and poor prognosis of cervical cancer. J Gynecol Oncol 28: e75, 2017.

61. Zhou LL, Shen Y, Gong JM, Sun P and Sheng JH: MicroRNA-466 with tumor markers for cervical cancer screening. Oncotarget 8: 70821-70827, 2017.

62. Yu J, Wang Y, Dong R, Huang X, Ding S and Qiu H: Circulating microRNA-218 was reduced in cervical cancer and correlated with tumor invasion. J Cancer Res Clin Oncol 138: 671-674, 2012.

63. You W, Wang Y and Zheng J: Plasma miR-127 and miR-218 Might Serve as Potential Biomarkers for Cervical Cancer. Reprod Sci 22: 1037-1041, 2015.

64. Luo M, Shen D, Wang W and Xian J: Aberrant expression of microRNA-26b and its prognostic potential in human cervical cancer. Int J Clin Exp Pathol 8: 5542-5548, 2015.

65. Liu S, Pan X, Yang Q, Wen L, Jiang Y, Zhao Y and Li G: MicroRNA-18a enhances the radiosensitivity of cervical cancer cells by promoting radiation-induced apoptosis. Oncol Rep 33: 2853-2862, 2015

66. Xie H, Lee L, Scicluna P, Kavak E, Larsson C, Sandberg R and Lui WO: Novel functions and targets of miR-944 in human cervical cancer cells. Int J Cancer 136: E230-E241, 2015.

67. Shen S, Wang L, Jia Y, Hao Y, Zhang L and Wang H: Upregulation of microRNA-224 is associated with aggressive progression and poor prognosis in human cervical cancer. Diagn Pathol 8: 69, 2013.

68. Huang L, Lin JX, Yu YH, Zhang MY, Wang HY and Zheng M: Downregulation of Six MicroRNAs Is Associated with Advanced Stage, Lymph Node Metastasis and Poor Prognosis in Small Cell Carcinoma of the Cervix. PLoS One 7: e33762, 2012.

69. Xing AY, Wang B, Shi DB, Zhang XF, Gao C, He XQ, Liu WJ and Gao P: Deregulated expression of miR-145 in manifold human cancer cells. Exp Mol Pathol 95: 91-97, 2013.

70. Lee H, Kim KR, Cho NH, Hong SR, Jeong H, Kwon SY, Park KH, An HJ, Kim TH, Kim I, et al: MicroRNA expression profiling and Notch1 and Notch2 expression in minimal deviation adenocarcinoma of uterine cervix. World J Surg Oncol 12: 334, 2014.

71. Ivanov MK, Titov SE, Glushkov SA, Dzyubenko VV, Malek AV, Arkhangelskaya PA, Samsonov RB, Mikhetko AA, Bakhidze EV, Berlev IV and Kolesnikov NN: Detection of high-grade neoplasia in air-dried cervical PAP smears by a microRNA-based classifier. Oncol Rep 39: 1099-1111, 2018.

72. Yu X, Zhao W, Yang X, Wang Z and Hao M: miR-375 affects the proliferation, invasion, and apoptosis of HPV16-positive human cervical cancer cells by targeting IGF-1R. Int J Gynecol Cancer 26: 851-858, 2016.

73. Hao M, Zhao W, Zhang L, Wang H and Yang X: Low folate levels are associated with methylation-mediated transcriptional repression of miR-203 and miR-375 during cervical carcinogenesis. Oncol Lett 11: 3863-3869, 2016.

74. Nagamitsu Y, Nishi H, Sasaki T, Takaesu Y, Terauchi F and Isaka K: Profiling analysis of circulating microRNA expression in cervical cancer. Mol Clin Oncol 5: 189-194, 2016.

75. Kogo R, How C, Chaudary N, Bruce J, Shi W, Hill RP, Zahedi P, Yip KW and Liu FF: The microRNA-218 Survivin axis regulates migration, invasion, and lymph node metastasis in cervical cancer. Oncotarget 6: 1090-1100, 2015.

76. Lajer CB, Garnæs E, Friis-Hansen L, Norrild B, Therkildsen MH, Glud M, Rossing M, Lajer H, Svane D, Skotte L, et al: The role of miRNAs in human papilloma virus (HPV)-associated cancers: Bridging between HPV-related head and neck cancer and cervical cancer. Br J Cancer 106: 1526-1534, 2012. 
77. Huang YW, Kuo CT, Chen JH, Goodfellow PJ, Huang TH, Rader JS and Uyar DS: Hypermethylation of miR-203 in endometrial carcinomas. Gynecol Oncol 133: 340-345, 2014.

78. Myklebust MP, Bruland O, Fluge Ø, Skarstein A, Balteskard L and Dahl O: MicroRNA-15b is induced with E2F-controlled genes in HPV-related cancer. Br J Cancer 105: 1719-1725, 2011.

79. Sharma Saha S, Roy Chowdhury R, Mondal NR, Chakravarty B, Chatterjee T, Roy S and Sengupta S: Identification of genetic variation in the lncRNA HOTAIR associated with HPV16-related cervical cancer pathogenesis. Cell Oncol (Dordr) 39: 559-572, 2016.

80. Andersen CL, Jensen JL and Ørntoft TF: Normalization of real-time quantitative reverse transcription-PCR data: A model-based variance estimation approach to identify genes suited for normalization, applied to bladder and colon cancer data sets. Cancer Res 64: 5245-5250, 2004.

81. Livak KJ and Schmittgen TD: Analysis of relative gene expression data using real-time quantitative PCR and the 2(-Delta Delta C(T)) method. Methods 25: 402-408, 2001.

82. Rossi ED, Bizzarro T, Martini M, Capodimonti S, Sarti D, Cenci T, Bilotta M, Fadda G and Larocca LM: The evaluation of miRNAs on thyroid FNAC: The promising role of miR-375 in follicular neoplasms. Endocrine 54: 723-732, 2016.

83. Kottaridi C, Spathis A, Margari N, Koureas N, Terzakis E, Chrelias C, Pappas A, Bilirakis E, Pouliakis A, Panayiotides IJ and Karakitsos P: Evaluation analysis of miRNAs overexpression in liquid-based cytology endometrial samples. J Cancer 8: 2699-2703, 2017.

84. Barodawala SM, Chadha K, Kavishwar V, Murthy A and Shetye S: Cervical cancer screening by molecular Pap-transformation of gynecologic cytology. Diagn Cytopathol 47: 374-381, 2019.
85. Bonde J, Ejegod DM, Cuschieri K, Dillner J, Heideman DAM, Quint W, Pavon Ribas MA, Padalko E, Christiansen IK, Xu L and Arbyn M: The Valgent4 protocol: Robust analytical and clinical validation of $11 \mathrm{HPV}$ assays with genotyping on cervical samples collected in SurePath medium. J Clin Virol 108: 64-71, 2018.

86. Tota JE, Bentley J, Blake J, Coutlée F, Duggan MA, Ferenczy A, Franco EL, Fung-Kee-Fung M, Gotlieb W, Mayrand MH, et al: Approaches for triaging women who test positive for human papillomavirus in cervical cancer screening. Prev Med 98: 15-20, 2017.

87. Derveaux S, Vandesompele J and Hellemans J: How to do successful gene expression analysis using real-time PCR. Methods 50: 227-230, 2010.

88. Schmittgen TD and Livak KJ: Analyzing real-time PCR data by the comparative C(T) method. Nat Protoc 3: 1101-1108, 2008

89. Kiss T: Small nucleolar RNAs: An abundant group of noncoding RNAs with diverse cellular functions. Cell 109: 145-148, 2002.

90. Dieci G, Preti M and Montanini B: Eukaryotic snoRNAs: A paradigm for gene expression flexibility. Genomics 94: 83-88, 2009.

91. Zubillaga-Guerrero MI, Alarcón-Romero Ldel C, IlladesAguiar B, Flores-Alfaro E, Bermúdez-Morales VH, Deas J and Peralta-Zaragoza O: MicroRNA miR-16-1 regulates CCNE1 (cyclin E1) gene expression in human cervical cancer cells. Int J Clin Exp Med 8: 15999-16006, 2015.

(i) $(9)$ This work is licensed under a Creative Commons Attribution-NonCommercial-NoDerivatives 4.0 International (CC BY-NC-ND 4.0) License. 